\title{
Modulation of phosphatidylinositol 3-kinase signaling reduces intimal hyperplasia in aortocoronary saphenous vein grafts
}

Jonathan A. Hata, MD, ${ }^{* a}$ Jason A. Petrofski, MD, ${ }^{* a}$ Jacob N. Schroder, MD, ${ }^{a}$ Matthew L. Williams, MD, a,b Sarah H. Timberlake, BS, ${ }^{c}$ Anne Pippen, BS, ${ }^{c}$ Michael T. Corwin, MD, ${ }^{,}$Amy K. Solan, MS, andre Jakoi, BS, ${ }^{a}$ Thomas R. Gehrig, MD, ${ }^{a}$ Christopher D. Kontos, MD, ${ }^{c}$ and Carmelo A. Milano, MDa

From the Departments of Surgery ${ }^{\mathrm{a}}$ and Medicine, ${ }^{\mathrm{c}}$ Duke University Medical Center, Durham, NC, and the Department of Surgery, ${ }^{\text {b }}$ Massachusetts General Hospital, Boston, Mass.

Supported in part by National Institutes of Health grant 1 RO1 HL072183-01 A1 and NRSA 5F32H71387-2.

Read at the Eighty-fourth Annual Meeting of The American Association for Thoracic Surgery, Toronto, Ontario, Canada, April 25-28, 2004.

Received for publication May 25, 2004; revisions received Oct 17, 2004; accepted for publication Nov 4, 2004.

Address for reprints: Carmelo A. Milano, MD, Box 304, Department of Surgery, Duke University Medical Center, Durham, NC 27703 (E-mail: milan002@mc.duke. edu)

*Drs Hata and Petrofski contributed equally to this work.

J Thorac Cardiovasc Surg 2005;129:1405-13

$0022-5223 / \$ 30.00$

Copyright (c) 2005 by The American Association for Thoracic Surgery

doi:10.1016/j.jtcvs.2004.11.048
Objectives: Fifty percent of human aortocoronary saphenous vein grafts are occluded after 10 years. Intimal hyperplasia is an initial step in graft occlusion and consists of vascular smooth muscle cell proliferation. Phosphatidylinositol 3-kinase and its downstream regulator, the inositol 3-phosphatase PTEN (phosphatase and tensin homolog deleted on chromosome 10), are important regulators of vascular smooth muscle cell proliferation, migration, and cell death. This study tests whether overexpression of PTEN in aortocoronary saphenous vein grafts can reduce intimal hyperplasia.

Methods: Adult dogs underwent aortocoronary bypass grafting to the left anterior descending artery by using the autologous saphenous vein. Saphenous vein grafts were treated with phosphate-buffered saline $(\mathrm{n}=9)$, empty adenovirus $(\mathrm{n}=8)$, or adenovirus encoding for PTEN $(n=8)$. Arteriography at 30 and 90 days assessed saphenous vein graft patency. A subset received saphenous vein grafts treated with a marker transgene ( $\beta$-galactosidase, $\mathrm{n}=3$ ), empty adenovirus $(\mathrm{n}=4)$, or adenovirus encoding for PTEN $(n=4)$ and were killed on postoperative day 3 to confirm expression. Vascular smooth muscle cells were isolated from canine saphenous vein infected with adenovirus encoding for PTEN, and immunoblotting and proliferation assays were performed.

Results: Saphenous vein graft transgene expression was confirmed by means of immunohistochemistry, immunoblotting, and polymerase chain reaction. Arteriograms revealed all saphenous vein grafts to be patent. Saphenous vein grafts treated with adenovirus encoding for PTEN demonstrated reduced intimal area compared with those treated with empty adenovirus and phosphate-buffered saline $\left(1.39 \pm 0.11\right.$ vs $2.35 \pm 0.3$ and $\left.2.57 \pm 0.4 \mathrm{~mm}^{2}, P<.05\right)$, and the intima/media ratio was lower in saphenous vein grafts treated with adenovirus encoding for PTEN $(0.50 \pm 0.05$ vs $1.43 \pm 0.18$ and $1.11 \pm 0.14, P<.005)$. PTEN overexpression in vascular smooth muscle cells inhibited platelet-derived growth factor-induced phosphorylation of Akt, a downstream effector of phosphatidylinositol 3-kinase. PTEN-treated vascular smooth muscle cells demonstrated decreased basal, platelet-derived growth factor-stimulated, and serumstimulated proliferation.

Conclusion: This study demonstrates that PTEN overexpression in aortocoronary saphenous vein grafts reduces intimal hyperplasia. The mechanism of this antiproliferative effect in vascular smooth muscle cells is likely due to inhibition of phosphatidylinositol 3-kinase signaling through Akt, with resultant decreases in vascular smooth muscle cell growth and survival. Therefore modulation of the phosphatidylinositol 3-kinase pathway through PTEN overexpression might represent a novel therapy to prevent saphenous vein graft intimal hyperplasia after coronary artery bypass grafting. 
$\mathrm{S}$ aphenous vein grafts (SVGs) represent the most common conduit used for surgical revascularization procedures, including coronary artery bypass grafting (CABG). Unfortunately, long-term aortocoronary SVG efficacy is limited by intimal hyperplasia (IH) and subsequent accelerated atherosclerosis, resulting in a 10-year graft failure rate approaching 50\%. ${ }^{1}$ Current attempts to limit SVG stenosis include technical considerations, antiplatelet therapy, and lipid-lowering medications. ${ }^{2,3}$ Despite these interventions, SVG failure after CABG remains a difficult problem, leading to recurrent angina and a $10 \%$ to $15 \%$ incidence of redo $\mathrm{CABG}$.

IH begins early after vein graft implantation and can eventually lead to luminal stenosis and occlusion. IH is characterized by abnormal migration of vascular smooth muscle cells (VSMCs) from the media to the intima. VSMCs subsequently proliferate and undergo hypertrophy, with associated deposition of an extracellular connective tissue matrix. ${ }^{4}$ Although not completely characterized, these pathologic changes are caused by the release of mitogenic growth factors in the setting of vascular injury..$^{5}$ Many growth factors and hormones can trigger IH by activating phosphatidylinositol (PI) 3-kinase in VSMCs. PI 3-kinase is a lipid kinase that phosphorylates phosphatidylinositol at the D-3 position of the inositol ring, and the resulting products, PI 3,4-bisphosphate (PI 3,4-P2) and PI 3,4,5trisphosphate (PIP3), are potent signaling molecules that regulate cell proliferation, migration, and survival. ${ }^{6} \mathrm{Re}-$ cently, the drug sirolimus (rapamycin), which inhibits a downstream effector of PI 3-kinase (mammalian target of rapamycin $[\mathrm{mTOR}]$ ), has shown promising results in the prevention of in-stent restenosis. ${ }^{7}$ In addition to mTOR, however, PI 3-kinase activates many other effectors, such as extracellular signal-regulated kinase (ERK) and Akt, which associate with membrane-bound 3-phosphoinositides and have been implicated as key mediators of cell growth, proliferation, and survival. ${ }^{8}$ Taken together, these studies implicate PI 3-kinase as a potential upstream regulator of these processes, leading to the hypothesis that inhibition of PI 3-kinase signaling might produce potent effects on IH.

Recent studies have investigated this hypothesis by using an endogenous inhibitor of PI 3-kinase in VSMCs. ${ }^{9,10}$ PTEN (phosphatase and tensin homolog deleted on chromosome 10) is an inositol 3-phosphatase that was originally characterized as a tumor suppressor protein. PTEN hydrolyzes the 3-phosphoinositide lipid products of PI 3-kinase, PI 3,4-P2, and PIP3, preventing downstream activation of PI 3-kinase effector molecules. Adenovirus-mediated expression of PTEN in rabbit VSMCs inhibited platelet-derived growth factor (PDGF)-induced cellular proliferation, migration, and survival, suggesting that in vivo overexpression of PTEN might prevent the formation of $\mathrm{IH}^{9}$
Although these initial studies represent important first steps, the effectiveness of PTEN to inhibit SVG IH has not yet been examined in a clinically relevant, large-animal model. Previously, we characterized a canine model of aortocoronary SVG IH and demonstrated efficient transgene expression by using ex vivo delivery of adenoviral vectors. ${ }^{11}$ This study tests the hypothesis that treatment of aortocoronary SVGs with an adenoviral vector encoding the PTEN transgene can limit IH. Additionally, we investigate the effects of PTEN overexpression in cultured canine saphenous vein (SV) smooth muscle cells.

\section{Methods \\ Experimental Design}

Animals used in this study were mongrel $\operatorname{dogs}(\mathrm{n}=36$; weight, 27-32 kg). As previously described, each dog underwent CABG with a reversed SVG from the aorta to the left anterior descending artery (LAD). ${ }^{11}$ Before grafting, veins were treated with $5 \times 10^{11}$ total virus particles of either an adenovirus encoding for PTEN (AdPTEN; $\mathrm{n}=8$ ) or empty adenovirus (AdEV; $\mathrm{n}=8$ ). A third group of dogs were sham-infected by means of treatment of the SVG with phosphate-buffered saline (PBS; $n=9$ ). SVG patency was confirmed by arteriograms performed on postoperative days (PODs) 30 and 90. Dogs were subsequently euthanized, and the SVGs were harvested.

A subset of dogs received SVGs treated with the marker transgene $\beta$-galactosidase (Ad $\beta$ gal; $\mathrm{n}=3$ ) and were killed on POD 3 to confirm transgene expression. Another subset of dogs treated with AdPTEN $(n=4)$ or $\operatorname{AdEV}(n=4)$ was killed on POD 3 for histologic analysis.

All animal procedures were humanely performed in accordance with regulations adopted by the National Institutes of Health and approved by the Animal Care and Use Committee of Duke University.

\section{Adenovirus Construction}

The structure of a recombinant, replication-deficient adenovirus directing the expression of wild-type human PTEN (AdPTEN) has been described previously. ${ }^{10}$ Control viruses were also constructed, including an empty adenovirus containing no cDNA insert (AdEV), and adenoviruses containing the coding sequence for $\beta$-galactosidase (Ad $\beta$ gal) and the green fluorescent protein (AdGFP). Adenoviruses were amplified on HEK-293 cells and purified by means of ultracentrifugation on a $\mathrm{CsCl}$ density gradient, as previously described. ${ }^{10}$ The procedures and handling of animals and tissues exposed to recombinant adenovirus were approved by the Institutional Biosafety Committee of Duke University in compliance with guidelines from the National Institutes of Health.

\section{Operative Protocol}

SV harvest, SVG gene delivery, and CABG were performed as previously described. ${ }^{11}$ Briefly, each dog was sedated, intubated, and heparinized (100 U/kg). Ten centimeters of SV was harvested from the hind leg. For the AdPTEN- or AdEV-treated groups, $5 \times 10^{11}$ total virus particles were suspended in $1 \mathrm{~mL}$ of PBS. The adenoviral solution was delivered to the SV 
intraluminally with a measured distension pressure of $10 \mathrm{~mm}$ $\mathrm{Hg}$ for 20 to 30 minutes. For the PBS group, the SV was flushed only. A partial lower sternotomy was performed, and an endto-side anastomosis was performed between the SVG and the ascending aorta. By using a myocardial stabilizer (Guidant), an end-to-side anastomosis was performed between the SVG and the distal LAD. The proximal LAD was subsequently ligated, rendering the anterior left ventricle SVG-dependent. An ultrasonic vascular probe (Transonic Systems) confirmed SVG flow. Dogs were maintained on buffered aspirin $(325 \mathrm{mg} / \mathrm{d})$ throughout the protocol.

\section{Arteriographic Confirmation of SVG Patency}

On PODs 30 and 90, the dogs underwent coronary arteriography. A $6 \mathrm{~F}$ coronary catheter was placed into the left coronary artery under fluoroscopic guidance, and radio-opaque dye was used to confirm the patency of the SVG.

\section{Tissue Preparation and Histologic Analysis}

At the time of death, tissues were harvested, including SVGs, nongrafted SV, left and right ventricles, livers, and lungs. Hematoxylin and eosin and X-gal staining were performed using standard techniques. ${ }^{12}$ An anti-PTEN monoclonal antibody (clone A2B1, Santa Cruz Biotech) was used to immunostain for PTEN expression.

In a subset of $\operatorname{dogs}(\mathrm{n}=3)$, short segments of AdPTENinfected SV were cultured for 3 days, and PTEN expression was assessed by means of immunoblotting.

\section{Morphologic Analysis}

Five-micrometer transverse vessel sections were stained with hematoxylin and eosin, and measurements were made with Image Tool version 3.0 (The University of Texas Health Sciences Center, San Antonio), as previously described. ${ }^{11}$ For each $\mathrm{dog}, 3$ sections from each third of the SVG were analyzed, and the intimal area, medial area, and maximal-minimal wall thickness were quantified. The ratio of the intimal to meldial area was calculated.

\section{DNA Isolation and PCR}

Tissue was incubated in lysis buffer $(100 \mathrm{mmol} / \mathrm{L}$ Tris/ $\mathrm{HCl}, 5$ $\mathrm{mmol} / \mathrm{L}$ ethylenediamine tetraacetic acid, $0.2 \%$ sodium dodecylsulfate, $200 \mathrm{mmol} / \mathrm{L} \mathrm{NaCl}$, and $0.2 \mathrm{mg} / \mathrm{mL}$ Proteinase K [Sigma]). The supernatant was extracted, and the DNA was precipitated. A polymerase chain reaction (PCR) was performed using a forward primer specific for the cytomegalovirus promoter and a reverse primer specific for human PTEN.

\section{Cell Harvest and Culture}

VSMCs were isolated from canine SVs harvested under sterile conditions. Briefly, the adventitia was stripped away, and the intima was removed by means of blunt dissection. The media was cut into $1-\mathrm{cm}^{2}$ sections and placed in culture dishes containing a small amount of growth medium, as previously described. ${ }^{13}$ After 10 days, veins were removed, and monolayers of smooth muscle cells were trypsinized and passaged. In this study, only cells between passages 3 and 5 were used.

\section{Adenovirus Infection}

VSMCs were grown in Dulbecco's modified Eagle's medium/ F12 HAM containing 10\% fetal bovine serum (FBS). When the cells were nearly confluent, the medium was changed to $2 \%$ FBS, and virus vectors were added at a multiplicity of infection of 100. After 24 hours, the medium was changed to serum-free medium, followed by different treatments or stimuli as indicated. As a control in all experiments, an identical group of cells was left uninfected.

\section{Immunoblotting}

After infection with adenoviruses, VSMCs were serum starved for 5 hours and then stimulated for 5 minutes with PDGF $(20 \mathrm{ng} / \mathrm{mL}$, R\&D Systems). Cells were lysed, and samples were separated by means of sodium dodecylsulfate $8 \%$ to $16 \%$ polyacrylamide gel electrophoresis. ${ }^{14}$ Membranes were Western blotted with the following antibodies: anti-PTEN monoclonal antibody (clone A2B1, Santa Cruz Biotech); anti-phospho-Akt (Ser473), anti-Akt, antiphospho-p44/42 ERK (Thr202/Tyr204), and anti-p44/42 ERK (Cell Signaling Tech); and rat monoclonal anti- $\alpha$-tubulin (clone YL1/2, Abcam).

\section{Thymidine Incorporation and Cell Counts}

Tritiated thymidine incorporation was assayed, as previously described, ${ }^{10}$ to evaluate the effect of PTEN overexpression on PDGF-mediated DNA synthesis in VSMCs. Briefly, VSMCs were plated and either left uninfected or infected with AdPTEN or AdGFP. The cells were pulse labeled with tritiated thymidine (2 $\mu \mathrm{Ci} / \mathrm{mL}$, Amersham-Pharmacia Biotech) for 3 hours, and thymidine incorporation was assessed by means of liquid scintillation counting.

For cell counts, VSMCs were plated in triplicate on 12-well plates and either left uninfected or infected with AdPTEN or AdGFP. The medium was changed to serum-free medium, and the cells were incubated for 48 hours with or without PDGF (20 $\mathrm{ng} / \mathrm{mL}$ ) or FBS (5\%). The cells were trypsinized and counted on a hemacytometer (Fisher).

\section{Statistical Analysis}

All data are represented as the mean \pm standard error of the mean. Statistical significance was determined by means of 1-way analysis of variance. Pairwise comparisons were then made with the Bonferroni or Student-Newman-Keuls post-hoc tests.

\section{Results}

Assessment of SVG Patency

SVGs from all dogs in this study $(\mathrm{n}=36)$ were confirmed to be patent immediately after completion of the distal anastomosis with an ultrasonic vascular flow probe. All flows were greater than $25 \mathrm{~mL} / \mathrm{min}$. Two dogs (both control group) died within 72 hours postoperatively and were excluded from the study. All of the remaining dogs in the 90-day cohort $(\mathrm{n}=25)$ survived to POD 90 and were included in the analysis. Arteriograms obtained on PODs 30 and 90 demonstrated all SVGs to be patent. Qualitative analysis revealed extensive filling defects consistent with graft wall thickening throughout the PBS and AdEV-treated 
SVGs. AdPTEN- treated SVGs did not demonstrate severe defects (data not shown).

\section{Assessment of Transgene Expression}

In Ad $\beta$ gal- and AdPTEN-treated SVGs explanted on POD 3 , transgene expression was confirmed on the basis of histologic staining (Figure 1, A-D). Transgene expression was robust and circumferential in the intima, with more diffuse expression throughout the media. In SVs undergoing adenoviral infection followed by 3 days of in vitro culture, Western blotting revealed marked PTEN expression compared with that seen in uninfected veins (data not shown).

PCR analysis was performed on total DNA isolated from SVG, normal SV, left and right ventricular myocardium, liver, and lung samples from AdPTEN-treated dogs killed on POD $3(n=4)$. The primers used were specific for the adenoviral PTEN transgene. The PTEN transgene DNA was detected in all SVG samples, and normal SVs from the same dogs served as negative controls (Figure 1,E). Similar tissue was harvested from AdEV-treated dogs $(n=4)$ and demonstrated no evidence of PTEN transgene expression (Figure 1, E). Additionally, left and right ventricular myocardium, liver, and lung samples from the AdPTEN-treated dogs demonstrated no evidence of transgene expression (data not shown).

\section{Quantification of Intimal Hyperplasia}

AdPTEN-treated SVGs demonstrated reduced intimal area compared with AdEV- and PBS-treated grafts (1.39 \pm 0.11 vs $2.35 \pm 0.3$ and $2.57 \pm 0.4 \mathrm{~mm}^{2} ; P<.05$, analysis of variance with Student-Newman-Keuls test; Figure 2 and Table 1). Additionally, the intima/media ratio was lower in AdPTEN-treated SVGs compared with that in AdEV- and PBS-treated grafts $(0.50 \pm 0.05$ vs $1.43 \pm 0.18$ and $1.11 \pm$ $0.14, P<.005)$. Medial area and maximum-minimum wall thickness were not significantly different among groups. Representative photomicrographs of SVGs from each group and a normal SV are shown in Figure 2.

\section{VSMC Signaling and Proliferation}

VSMCs were cultured from canine SVs and infected with recombinant adenoviruses expressing wild-type PTEN (AdPTEN) or GFP (AdGFP) to investigate the mechanism of action of AdPTEN in SVGs. As an additional control, uninfected cells were evaluated in an identical manner. Cells were then treated with PDGF to induce cellular proliferation. Although PTEN is expressed endogenously in canine VSMCs, AdPTEN infection induced significant overexpression (Figure 3). Treatment of canine uninfected or AdGFP-infected VSMCs with PDGF induced phosphorylation of Akt, which correlates with Akt activation (Figure 3). Overexpression of PTEN blocked PDGF-induced phosphorylation of Akt in VSMCs (Figure 3). Likewise, PDGF stimulation of uninfected or AdGFP-infected VSMCs increased phosphorylation of ERK. However, overexpression of PTEN did not inhibit ERK phosphorylation. These results suggest that the effects of PTEN on VSMC growth are mediated in part by inhibition of Akt but not ERK.

We next investigated whether the effects of PTEN overexpression on PI 3-kinase-mediated signaling pathways correlated with cellular responses relevant to the process of IH. Canine SV VSMCs were infected with AdPTEN, AdGFP, or left uninfected. PDGF- and FBS-stimulated DNA synthesis was measured by means of tritiated thymidine incorporation. PDGF induced significant increases in DNA synthesis in uninfected and AdGFP-infected VSMCs (Figure 4, A). PTEN overexpression significantly decreased basal thymidine incorporation, as well as in response to PDGF and FBS (Figure 4, $A$ ).

Cell counts were performed on uninfected and AdGFPor AdPTEN-infected VSMCs treated with or without PDGF or FBS to confirm these findings. Both PDGF and FBS induced a significant increase in the number of uninfected or AdGFP-infected canine VSMCs (Figure 4, B). PTEN overexpression significantly decreased the number of unstimulated cells compared with AdGFP-infected or uninfected cells. PTEN overexpression also inhibited both PDGF- and FBS-stimulated increases in cell number (Figure 4, B). Together, these findings demonstrate that PTEN overexpression blocks PDGF- and FBS-mediated increases in VSMC proliferation.

\section{Discussion}

Pathologic VSMC proliferation and associated IH is a critical early factor leading to aortocoronary SVG failure. ${ }^{15}$ PI 3 -kinase plays a central role in this process through its 3-phosphoinositide lipid products, PI $(3,4) \mathrm{P} 2$ and PIP3, which regulate the activation of downstream effector molecules, including Akt and mTOR. ${ }^{6}$ Specifically, Akt is recruited to and associates with the plasma membrane-bound 3-phosphoinositides. Akt subsequently activates mTOR, which in turn phosphorylates key ribosomal proteins involved in cell protein translation. ${ }^{14}$ As a result, hydrolysis of PI $(3,4)$ P2 and PIP3 by PTEN, an inositol phosphatase specific for these lipid products, would be expected to inhibit the cellular processes required for IH.

In this study we tested the effects of inhibiting PI 3-kinase-mediated signaling by overexpressing the inositol 3-phosphatase PTEN through adenovirus-mediated gene transfer in a large-animal model of aortocoronary SVG IH. Indeed, SVGs treated with AdPTEN demonstrated an approximately $50 \%$ reduction in intimal area relative to saline and AdEV-infected control SVGs 90 days after grafting. This was accompanied by a significant decrease in the intima/media ratio. PTEN overexpression in vitro in canine SV VSMCs led to inhibition of an important effector of PI 

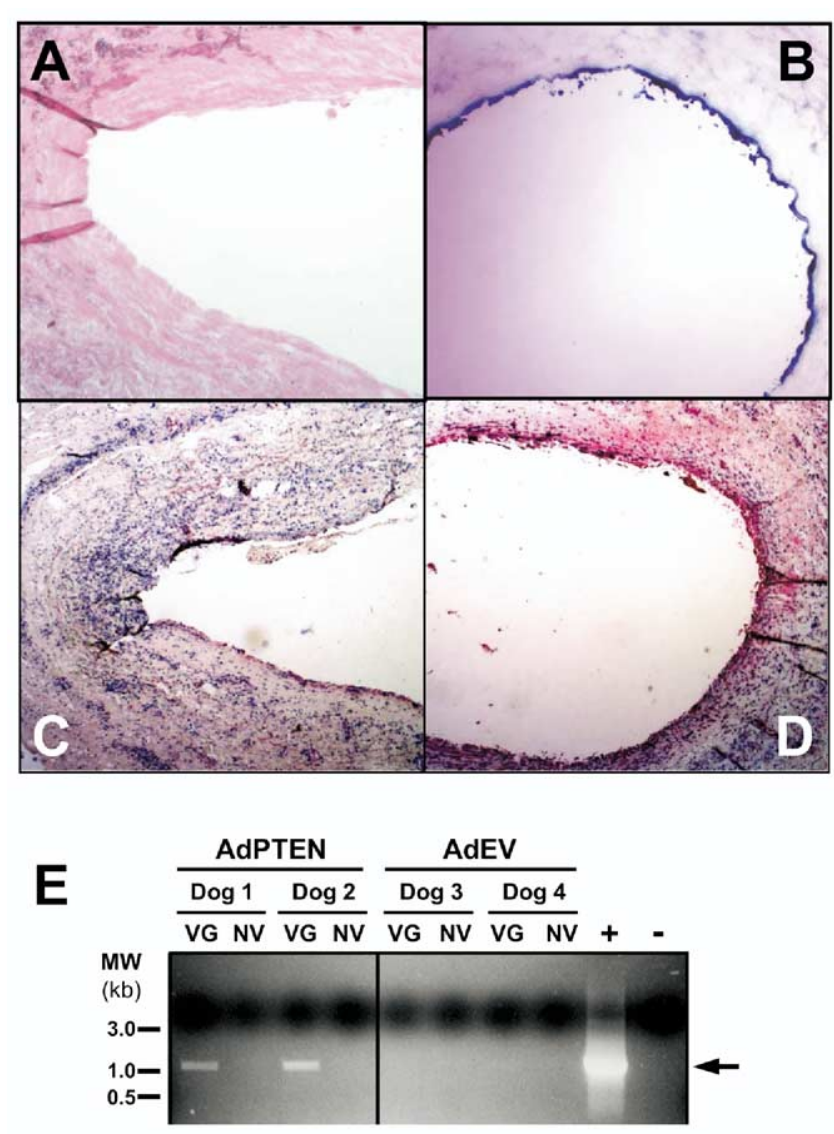

Figure 1. Adenovirus-mediated overexpression of transgenes in canine aortocoronary SVGs. Representative sections (10 $\mu \mathrm{m})$ from SVGs explanted on POD 3 are shown. A, Uninfected control (Xgal stain); B, Ad $\beta$ gal-infected (X-gal stain, $\beta$ gal = blue); C, AdEV-infected (anti-PTEN stain, PTEN = red); and D, AdPTENinfected (anti-PTEN stain, PTEN = red). E, PCR analysis performed on SVGs explanted on POD 3: expression of the PTEN transgene in AdPTEN- or AdEV-infected SVGs (VG) versus normal vein (NV). +, Positive control; -, negative control.

3-kinase, Akt. Moreover, PTEN overexpression significantly inhibited both basal and PDGF-induced VSMC proliferation. Together, these results suggest that PTEN overexpression might have a therapeutic role in preventing $\mathrm{IH}$ after vascular interventions, such as CABG.

The canine model of aortocoronary SVG IH used in this study was first characterized by Brody and colleagues ${ }^{16,17}$ and Silver and associates. ${ }^{18}$ These studies demonstrated that histologic changes closely resembling those seen in human aortocoronary SVGs, including medial fibrosis and IH, occurred within the first month after surgical intervention. We further characterized this canine model and demonstrated that by POD 90, the intimal area and the intima/media ratio were dramatically increased in SVGs compared with un-

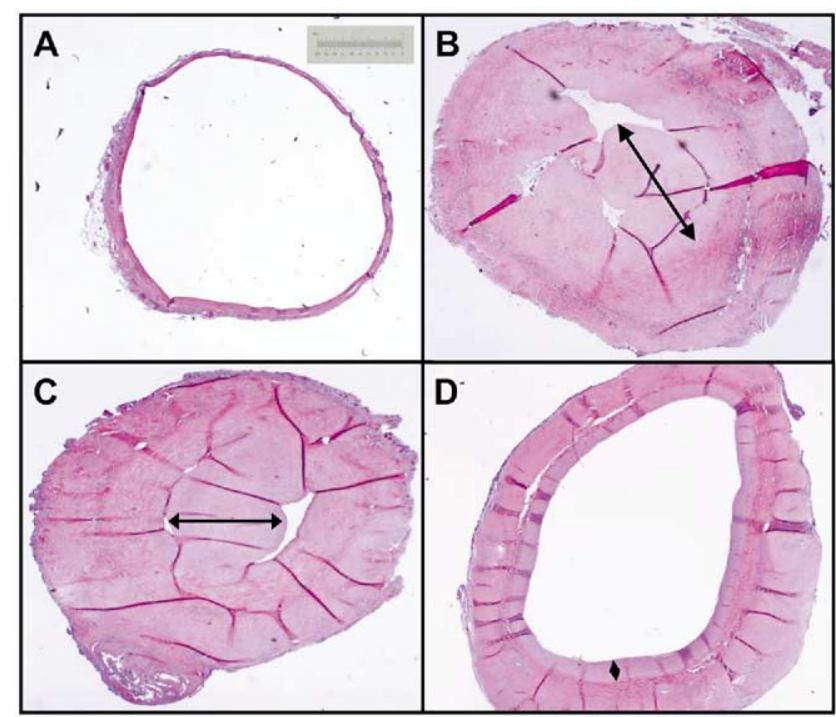

Figure 2. PTEN overexpression inhibits IH. Representative hematoxylin and eosin-stained sections of normal SVG (A), PBStreated SVG (B), AdEV-treated SVG (C), and AdPTEN-treated SVG (D) from animals killed on POD 90. Arrows indicate intimal borders (scale $=1 \mathrm{~mm}$ ).

grafted veins. ${ }^{11}$ Furthermore, careful histologic analysis of the vein grafts demonstrated that the IH occurred along the entire length of the grafted vessel. Although this model does not completely mimic human vein graft hyperplasia in that it lacks underlying atherosclerotic accumulation of foam cells or lipid deposits, we believe it represents the most relevant preclinical model in which to test potential therapies. Appropriate caution should be exercised, however, in extrapolating the results of this relatively short-term, largeanimal model to the chronic, multifactorial disease process that occurs in human SVG failure.

We and other groups have demonstrated that an ex vivo adenoviral delivery technique can produce efficient transgene expression in aortocoronary SVGs. ${ }^{11,19}$ The current study used this technique to successfully deliver a potentially therapeutic transgene to aortocoronary SVGs, resulting in dramatic inhibition of IH. In addition to PTEN, several other targets have shown promise for the prevention of IH. Cable and coworkers ${ }^{20}$ demonstrated that adenovirusmediated overexpression of nitric oxide synthase significantly reduced $\mathrm{IH}$ in cultured human SVGs. Similar results were obtained after overexpression of tissue inhibitors of matrix metalloproteinases. ${ }^{21}$ Using a nonviral delivery method, Shitani and colleagues ${ }^{22}$ demonstrated that pressure-mediated transfection of decoy oligonucleotides of nuclear factor $\kappa \mathrm{B}$ resulted in decreased $\mathrm{IH}$ after 4 weeks in a canine aortocoronary SVG model similar to that used in this study. Most importantly, the efficacy of E2F decoy oligo- 
TABLE 1. Morphologic analysis of canine SVGs 90 days after CABG

\begin{tabular}{|c|c|c|c|c|}
\hline & Saline $(n=9)$ & $E V(n=8)$ & AdPTEN $(n=8)$ & $P$ value $^{*}$ \\
\hline Intimal area $\left(\mathrm{mm}^{2}\right)$ & $2.57 \pm 0.4$ & $2.53 \pm 0.3$ & $1.39 \pm 0.11$ & $<.05$ \\
\hline Medial area $\left(\mathrm{mm}^{2}\right)$ & $2.28 \pm 0.22$ & $1.95 \pm 0.48$ & $2.8 \pm 0.13$ & NS \\
\hline $\mathrm{I} / \mathrm{M}$ ratio & $1.11 \pm 0.14$ & $1.43 \pm 0.18$ & $0.50 \pm 0.05$ & $<.005$ \\
\hline Maximum wall thickness (mm) & $1.64 \pm 0.29$ & $1.42 \pm 0.15$ & $1.16 \pm 0.06$ & NS \\
\hline Minimum wall thickness (mm) & $0.65 \pm 0.06$ & $0.72 \pm 0.07$ & $0.62 \pm 0.03$ & NS \\
\hline
\end{tabular}

Saphenous vein grafts were treated ex vivo with adenovirus encoding for PTEN ( $5 \times 10^{11}$ particles) versus empty virus or saline controls. EV, Empty virus; AdPTEN, adenovirus encoding for PTEN; I/M ratio, intimal/medial ratio. *P value: adenovirus encoding for PTEN versus saline and empty virus (analysis of variance ([Student-Newman-Keuls test]).

nucleotides to reduce the rate of peripheral SVG failure ${ }^{23}$ resulted in the first clinical trial of human aortocoronary SVG gene therapy (PREVENT IV), which is currently underway. Taken together, these studies suggest that SVG IH is a multifactorial process that has yet to be fully characterized and for which several therapies in combination might prove to be the most efficacious.

The results of this study highlight several issues related to extending adenovirus-mediated delivery techniques to clinical vascular gene therapy. Although significant inhibition of IH was observed up to 90 days postoperatively, the typical duration of transgene expression with first-generation adenoviral vectors, such as that used here, is typically

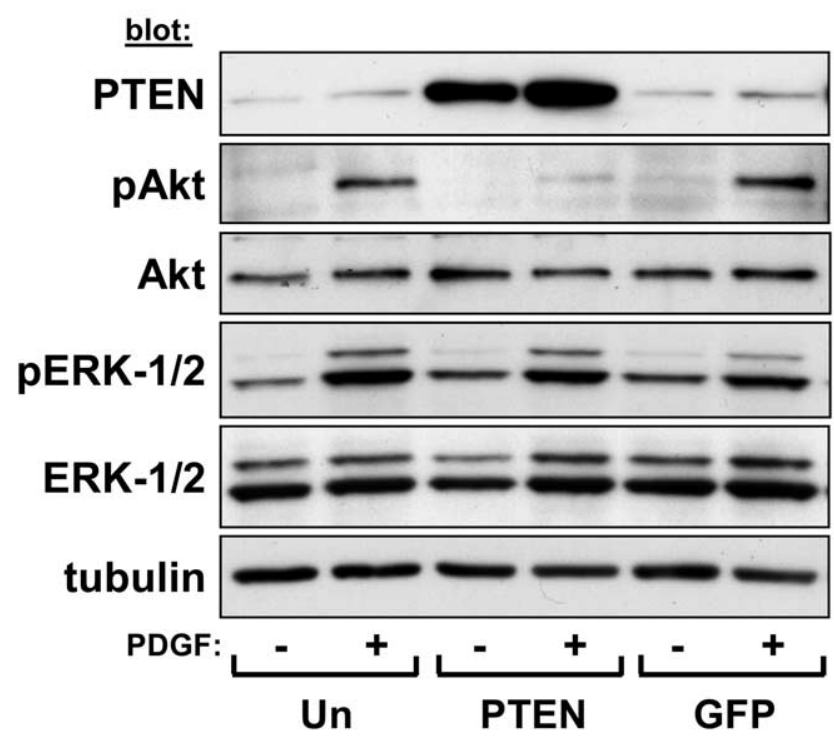

Figure 3. Overexpression of PTEN inhibits Akt phosphorylation in canine SV VSMCs. Lysates from VSMCs infected with the indicated adenoviruses and treated with or without PDGF $(20 \mathrm{ng} / \mathrm{mL})$ for 5 minutes were Western blotted sequentially with antibodies specific for PTEN, phospho-Akt (pAkt), and phospho-ERK (pERK). Equal protein loading was confirmed with antibodies against total Akt, total ERK, and $\alpha$-tubulin. Similar results were observed in 6 different experiments. Un, Uninfected; GFP, green fluorescent protein. less than 3 weeks. This suggests that inhibition of molecular triggering mechanisms early after vascular injury might be sufficient to produce intermediate or long-term beneficial effects. A potential explanation for this effect is that inhibition of VSMC proliferation is required only until the damaged vessel endothelium can be regenerated. If this hypothesis is true, adenoviral vectors might be ideal for vascular gene therapy because their limited length of action would avoid the possibility of long-term toxicity. This is especially relevant to transgenes with a proapoptotic mechanism, such as PTEN, in which chronic expression might prove deleterious. Additionally, future studies need to be performed to assess the efficacy of this method in preventing long-term IH. Clearly, the robust IH seen in this canine model occurs more rapidly than that typically seen in human subjects, and although the reasons for this remain unclear, it represents a major limitation of this study. However, the ability of transgenes such as PTEN to attenuate IH up to 3 months postoperatively suggests that such techniques might hold promise for the clinical arena.

Another important advantage of potential clinical vascular gene therapy is local delivery. In this study the adenovirus is delivered with an ex vivo method confined solely to the vessel and occurring during the natural course of a bypass operation. As expected with this technique, PTEN transgene expression limited to the SVG was not observed in any other tissues, including left ventricular myocardium perfused by the implanted graft. Treatment of harvested or artificial vascular conduits before implantation might represent the most safe and clinically applicable approach with which to attempt human vascular gene therapy.

In this study transgene expression in the SVGs was assessed using several different methods, including immunoblotting, histologic staining, and PCR. To initially confirm the expression of PTEN using the ex vivo delivery method, we performed immunoblotting on infected SVGs after 3 days of in vitro culture (data not shown). In the immunostained sections of AdEV-infected veins, low levels of endogenous PTEN were visible, and endogenous PTEN was readily detectable in cultured canine VSMCs. PTEN is highly homologous across species, both at the DNA and 
protein levels. Because of the ubiquitous expression of PTEN throughout body tissues, we modified our PCR technique to achieve specificity for the adenoviral PTEN transgene. This was accomplished by using a forward primer specific for the adenoviral vector's cytomegalovirus promoter and a reverse primer specific for human PTEN, producing a very specific and sensitive test for the PTEN transgene.

The effect of PTEN on the molecular and cellular responses of VSMCs was evaluated in the presence of 2 stimulants, PDGF and FBS. As a smooth muscle growth factor, PDGF is a powerful VSMC mitogen and survival factor that is released during arterial injury and contributes to IH. ${ }^{24-26}$ In contrast, serum contains a host of other growth factors and hormones, which are also known to activate PI 3-kinase and its downstream effectors. The observed ability of PTEN to attenuate VSMC responses to both PDGF and FBS at a cellular level suggests a potential mechanism for the observed in vivo effects. However, there are undoubtedly many mechanisms involved in the process of SVG IH that do not involve smooth muscle cell inhibition and are therefore unaffected by PTEN overexpression.

It is likely that the observed inhibition of VSMC proliferation by PTEN was mediated in part by an increase in apoptosis, an effect that has been demonstrated previously in arterial VSMCs. ${ }^{9}$ Serum starvation induced only moderate cell loss in the canine VSMCs, whereas a significant decrease in cell number was observed in PTEN-expressing cells, even in the presence of PDGF or FBS. Accordingly, PTEN overexpression markedly inhibited activation of Akt, a critical regulator of cell survival. Thus we postulate that some of the antiproliferative effects of PTEN overexpression were due to enhanced cell death. Further studies, including direct measures of saphenous VSMC apoptosis, are needed to confirm this hypothesis.

Importantly, the proliferative response of AdPTEN-infected cells to PDGF or FBS was significantly inhibited, suggesting that the effects of PTEN were not solely due to apoptosis but that PTEN also blocked a specific proliferative pathway. PI 3-kinase signaling has been linked to cellular proliferation in a variety of cell types, which has been associated with activation of both ERK and Akt. ${ }^{27}$ PI 3-kinase has been shown to be either upstream or downstream of ERK and to be activated through a parallel or independent pathway. ${ }^{28,29}$ In this study overexpression of PTEN, which inhibits all PI 3-phosphoinositide-dependent signaling, inhibited activation of Akt but not ERK. This finding is consistent with previous results in endothelial and arterial VSMCs, in which PTEN overexpression did not alter ERK activation. ${ }^{9}$ These findings demonstrate that PTEN and PI 3-kinase are not upstream of ERK in the canine VSMCs used in this study.
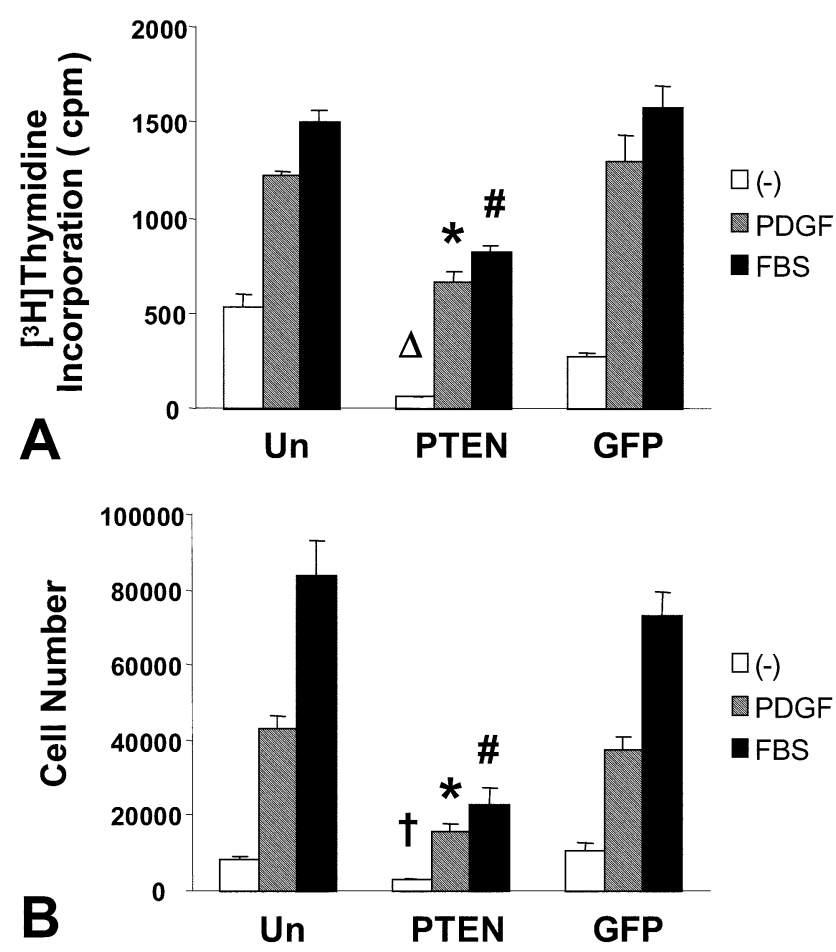

Figure 4. PTEN inhibits canine SV VSMC proliferation. A, VSMCs were either uninfected $(U n)$ or infected with AdPTEN or AdGFP, quiesced in serum-free medium for 24 hours, treated with or without PDGF (20 ng/mL) or FBS (5\%) for 18 hours, and then pulse labeled with tritiated thymidine. Thymidine incorporation was assessed by uninfected scintillation counting of precipitated DNA ( $\mathrm{n}=\mathbf{3}$ assays performed in triplicate). $\Delta P<.05$ versus uninfected and GFP unstimulated VSMCs (-); ${ }^{*}<.05$ versus uninfected and GFP PDGF-stimulated VSMCs; \#P $<.01$ versus uninfected and GFP FBS-stimulated VSMCs (analysis of variance, Bonferroni post-hoc test). B, VSMCs were treated as in A, except cells were grown for 48 hours in serum-free media or in the presence of PDGF or FBS. Then cells were trypsinized and counted ( $n=5$ assays performed in triplicate). $+\boldsymbol{P}<.05$ versus GFP-stimulated VSMCs $(-) ;{ }^{*}<.01$ versus uninfected or GFP PDGF-stimulated VSMCs; \#P $<.001$ versus uninfected or GFP FBS-stimulated VSMCs.

In addition to being activated by many types of cellular receptors, PI 3-kinase is ubiquitously expressed in tissues throughout the body. As a result, systemic inhibition of PI 3-kinase by PTEN would likely cause serious toxicity, potentially mimicking the chemotherapeutic effects of drugs such as rapamycin, which targets the PI 3-kinase downstream effector mTOR. However, just as local delivery of rapamycin in coated stents has yielded positive early results, local expression of a transgene such as PTEN might be clinically desirable. Indeed, in targeting the phospholipid products of PI 3-kinase, PTEN is proximal to effectors such as mTOR and Akt in this signaling pathway. Therefore 
PTEN overexpression should inhibit the entire cascade of downstream PI 3-kinase effectors, potentially yielding a more potent inhibitory effect on $\mathrm{IH}$.

\section{Conclusion}

This study demonstrates that adenovirus-mediated expression of PTEN inhibits aortocoronary SVG IH in a largeanimal model. This antiproliferative effect is mediated in canine and human VSMCs through inhibition of PI 3-kinase signaling. These results suggest that modulation of the PI 3-kinase pathway through PTEN overexpression might represent a novel therapy to prevent IH in vascular conduits, such as aortocoronary SVGs.

\section{Addendum}

A recently published study from our group ${ }^{30}$ demonstrated that adenovirus-mediated overexpression of PTEN in a carotid arterial injury model significantly inhibited neointimal hyperplasia, in part due to an increase in medial cell apoptosis. Together, these findings suggest that delivery of PTEN to the vessel wall may have similar effects in different vascular injury states, such as restenosis following percutaneous vascular interventions and CABG.

We thank George Quick, Ronald Johnson, Elaine Parker, and Mike Lowe for their valuable technical support during all animal procedures in this study and Damian Craig for statistical assistance.

\section{References}

1. Campeau L, Enjalbert M, Lesperance J, Vaislic C, Grondin CM, Bourassa MG. Atherosclerosis and late closure of aortocoronary saphenous vein grafts: sequential angiographic studies at 2 weeks, 1 year, 5 to 7 years, and 10 to 12 years after surgery. Circulation. 1983;68(suppl II):II1-7.

2. Souza DS, Dashwood MR, Tsui JC, et al. Improved patency in vein grafts harvested with surrounding tissue: results of a randomized study using three harvesting techniques. Ann Thorac Surg. 2002;73:1189-95.

3. Goldman S, Copeland J, Moritz T, et al. Improvement in early saphenous vein graft patency after coronary artery bypass surgery with antiplatelet therapy: results of a Veterans Administration Cooperative Study. Circulation. 1988;77:1324-32.

4. Gibbons GH, Dzau VJ. The emerging concept of vascular remodeling. N Engl J Med. 1994;330:1431-8.

5. Braun-Dullaeus RC, Mann MJ, Dzau VJ. Cell cycle progression: new therapeutic target for vascular proliferative disease. Circulation. 1998; 98:82-9.

6. Rameh LE, Cantley LC. The role of phosphoinositide 3-kinase lipid products in cell function. $J$ Biol Chem. 1999;274:8347-50.

7. Sousa JE, Costa MA, Abizaid AC, et al. Sustained suppression of neointimal proliferation by sirolimus-eluting stents: one-year angiographic and intravascular ultrasound follow-up. Circulation. 2001; 104:2007-11.

8. Downward J. Mechanisms and consequences of activation of protein kinase B/Akt. Curr Opin Cell Biol. 1998;10:262-7.

9. Huang J, Kontos CD. Inhibition of vascular smooth muscle cell proliferation, migration, and survival by the tumor suppressor protein PTEN. Arterioscler Thromb Vasc Biol. 2002;22:745-51.

10. Huang J, Kontos CD. PTEN modulates vascular endothelial growth factor mediated signaling and angiogenic effects. J Biol Chem. 2002; 277:10760-6.
11. Petrofski JA, Hata JA, Gehrig TR, et al. Gene delivery to aortocoronary saphenous vein grafts in a large animal model of intimal hyperplasia. J Thorac Cardiovasc Surg. 2004;127:27-33.

12. Shah AS, White DC, Tai O, et al. Adenovirus-mediated genetic manipulation of the myocardial $\beta$-adrenergic signaling system in transplanted hearts. J Thorac Cardiovasc Surg. 2000;123:581-8.

13. Gao J, Niklason L, Langer R. Surface hydrolysis of poly(glycolic acid) meshes increases the seeding density of vascular smooth muscle cells. J Biomed Mater Res. 1998;42:417-24.

14. Thomas G, Hall MN. TOR signaling and control of cell growth. Curr Opin Cell Biol. 1997;9:782-7.

15. Akowuah EF, Sheridan PJ, Cooper GJ, Newman C. Preventing saphenous vein graft failure: does gene therapy have a role? Ann Thorac Surg. 2003;76:L959-66.

16. Brody WR, Angell WW, Kosek JC. Histologic fate of the venous coronary artery bypass in dogs. Am J Pathol. 1972;66:111-30.

17. Brody WR, Kosek JC, Angell WW. Changes in vein grafts following aorto-coronary bypass induced by pressure and ischemia. $J$ Thorac Cardiovasc Surg. 1972;66:847-53.

18. Silver MD, Wilson GJ, Lixfield W, Trimble AS, MacGregor DC. Aortocoronary bypass graft in dogs: late histological changes. Pathology. 1976;8:343-51.

19. Chiu-Pinheiro CK, O'Brien T, Katusic ZS, Bonilla LF, Hammer CE, Schaff $\mathrm{H}$. Gene transfer to coronary artery bypass conduits. Ann Thorac Surg. 2002;74:1161-6.

20. Cable DG, Caccitolo JA, Caplice N, et al. The role of gene therapy for intimal hyperplasia of bypass grafts. Circulation. 1999;100(suppl II): II392-6.

21. George SJ, Boker AH, Angelini GD, et al. Gene transfer of tissue inhibitor of metalloproteinase-2 inhibits metalloproteinase activity and neointima formation in human saphenous veins. Gene Ther. 1998;5: 1552-60.

22. ShiNtani $\mathrm{T}$, Takahasi $\mathrm{T}$, Takahashi $\mathrm{T}$, et al. Intraoperative transfection of vein grafts with the $\mathrm{NF} \kappa \mathrm{B}$ decoy in a canine aortocoronary bypass model: a strategy to attenuate intimal hyperplasia. Ann Thorac Surg. 2002;74:1123-37.

23. Mann MK, Whittemore AD, Donaldson MC, et al. Ex-vivo gene therapy of human vascular bypass grafts with E2F decoy: the PREVENT single-centre, randomised, controlled trial. Lancet. 1999;354: 1493-8.

24. Walker LN, Bowen-Pope DF, Ross R, Reidy MA. Production of platelet-derived growth factor-like molecules by cultured arterial smooth muscle cells accompanies proliferation after arterial injury. Proc Natl Acad Sci U S A. 1986;83:7311-5.

25. Nabel EG, Yang Z, Liptay S, et al. Recombinant platelet-derived growth factor B gene expression in porcine arteries induce intimal hyperplasia in vivo. J Clin Invest. 1993;91:1822-9.

26. Heldin $\mathrm{CH}$, Westermark B. Mechanism and in vivo role of plateletderived growth factor. Physiol Rev. 1999;79:1283-316.

27. Pullen N, Thomas G. The modular phosphorylation and activation of p70s6k. FEBS Lett. 1997;410:78-82.

28. Xue L, Murray JH, Tolkovsky AM. The Ras/phosphatidylinositol 3-kinase, and Ras/ERK pathways that function as independent survival modules each of which inhibits a distinct apoptotic signaling pathway in sympathetic neurons. J Biol Chem. 2000;275:8817-24.

29. Nguyen KT, Wang WJ, Chan JL, Wang LH. Differential requirements of the MAP kinase and PI3 kinase signaling pathways in SRC- versus insulin and IGF-1 receptors-induced growth and transformation of rat intestinal epithelial cells. Oncogene. 2000;19:5385-97.

30. Huang J, Niu X-L, Pippen AM, Annex BH, Kontos CD. Adenovirusmediated intraarterial delivery of PTEN inhibits neointimal hyperplasia. Arterioscler Thromb Vasc Biol. 2005;25:354-8.

\section{Discussion}

Dr Michael Mann (San Francisco, Calif). First of all, I would like to commend you and your group on carrying the torch for vascular gene therapy in these dark days when most card-carrying gene therapists have to travel incognito, but I do have a couple of questions for you. 
PTEN, of course, being an intracellular enzymatic target, in this case for gene transfer, one would expect to depend on a very high, in this case, transduction efficiency or infection efficiency with the virus to hit a large population of the target smooth muscle cells that you are trying to inhibit. It looks like you very carefully reproduced previous data that had shown that the type of infection that you do is fairly effective at getting the intima, probably a lot of the endothelial cells, but not really very effective, probably less than $10 \%$, of the medial smooth muscle cells, which are actually your target.

I wonder whether you have thought about 2 things: (1) how you explain a measurable and even somewhat significant effect on IH through an intracellular target that allows you to only hit a very small percentage of your target cells and (2) whether you considered using alternative viruses, such as an adeno-associated virus, that at least in some people's hand has been better at getting across the internal elastic lamina?

Dr Hata. Thank you, Dr Mann, for your comments and questions. In terms of the intima versus media infectivity, you are absolutely correct, we would want to get infection of both layers, especially the VSMCs, before they are able to migrate into the intima and become the neointima. In our histologic sections of treated veins, we see expression in both layers. There is clearly robust expression in the intima, but we also see significant viral penetration into the media. As our results demonstrate, the resulting transgene expression in both layers is enough to effectively stop the migration and proliferation of VSMCs and the development of $\mathrm{IH}$.

In terms of other viruses that you mentioned, we are very interested, as most gene therapists are, in using improved vectors, because we believe this is currently one of the major limitations of the field. For example, we are in the process of obtaining and delivering adeno-associated viruses containing both marker genes and target transgenes, and those studies are ongoing.

Dr Robert C. Robbins (Stanford, Calif). You showed us nice data on cell proliferation and alluded to the potential of a mechanistic effect of this treatment on apoptosis, but you did not show us any data either in the article or in your presentation about apoptosis. What comment do you have about that?

Dr Hata. Prior studies by Kontos and associates have demonstrated that the action of PTEN in other types of VSMCs occurs in part by promoting apoptosis. Similar studies are ongoing to verify this mechanism in our model, both in vitro with isolated VSMCs and in the whole-vein sections. It will be interesting to see whether the results will be similar in our model of SVG IH to what has been demonstrated in other settings, such as arterial injury and restenosis.

Dr Robbins. When we have tried to look at IH in vein grafts, it is not like an artery that has an internal elastic membrane there, so what was your borderline for the IH in calculating your area and your intima/media ratio?

Dr Hata. I agree that it can be difficult to delineate each layer in the vein wall. On our sections we attempted to define the intima-media border as clearly as possible. In most sections we were able to determine where the circular muscle cells of the medial layer started and use that as our borderline.

Dr Ross M. Ungerleider (Portland, Ore). What do you predict would be the duration of this effect if you just pushed the time to proliferation off by several months and eventually we end up in the same place with this kind of treatment?

Dr Hata. An excellent question, and at this time, we do not know the answer. We examined the grafts after 90 days, which we had earlier demonstrated to be a reasonable period of time in this canine model for IH to develop. We have some dogs that we are keeping alive to 1 year, at which time we are going to perform similar arteriographic and histologic analyses. We do know that the transgene is only expressed for 2 or 3 weeks at most with this type of adenovirus. Therefore we hypothesize that an important part of the development of IH in these grafts is an early vascular injury event. The ability to block these early triggering mechanisms in this study led to inhibition of hyperplasia up to 90 days, but we hope to demonstrate in subsequent studies that this effect lasts even longer. 